International Journal of Engineering \& Technology, $7(1.3)(2018) 85-89$
International Journal of Engineering \& Technology
WPC
Website: $w$ ww.sciencepubco.com/index.php/IJET
Research paper

\title{
Implementation of boolean algebraic structure and its decision making approach over lattice ordered multisets
}

\author{
Anusuya Ilamathi V.S. ${ }^{1}$ and Vimala J. ${ }^{1^{*}}$ \\ ${ }^{1}$ Department of Mathematics, Alagappa University, Karaikudi, Tamilnadu India \\ *Corresponding author E-mail: vimaljey@alagappauniversity.ac.in
}

\begin{abstract}
A multiset is a collection of objects in which they are allowed to repeat. The purpose of this paper is to generalize the notion of Boolean algebra in the context of multisets. Furthermore, we consider 0 and 1 as multiset depiction and identify their role in Boolean algebra over lattice ordered multisets(dual), where some sorting exists among the parameters are explored.
\end{abstract}

Keywords: multiset;lattice ordered multisets; De Morgan algebra on multiset; Boolean algebra on multiset

\section{Introduction}

Multiset theory is an influential mathematical tool to handle unreliabilities. The crucial aspect of a multiset is the recurrence of its objects which we get it from a set and it is a new interesting mathematical notion. By way of illustration, repeated roots of polynomial equations, repeated elements in the prime factorization of an integer, repeated observations in statistical samples, repeated hydrogen atoms in a water molecule, a graph with loops, the various strands of the homogeneous DNA etc., need to be counted for obtaining adequacy and accuracy. The concept of multiset ensue repeated elements are admitted in a set. A categorical approach to multisets together with partially ordered multisets was explained in [11]. Weierstrass defines real numbers as certain msets of rational numbers in which finitely many repetitions are allowed. For example, the quantity $\pi=$ $3.141 \ldots .$. . can be identified with a multiset containing the number 1 with multiplicity 3 , the element $\frac{1}{10}$ with multiplicity 1 , the element $\frac{1}{100}$ with multiplicity 4 , etc.(see [15]).

Many other examples from daily life can be used to further illustrate these distinctions. Coins of the same denomination and year of minting but which are physically distinct are essentially indistinguishable so we consider them as same(equal) but not identical Electrons, or amino acids, or grains of sand, occur repeatedly in larger structures where their relative positions may allow us to distinguish them, although individually they may appear to us as the same, despite being obviously separate. Multiset theory is a splendid prospective applications in diverse circumstances; In those situations, recurrences of objects and its order become mandatory to the structure. For instance, employee position and their salary, price and sales of the product, syllabi and the centum scorers, disease speed and spread percentage. These circumstances give rise to an intention of lattice ordered multiset and its dual because number of students may decrease as percentage of obtained marks goes higher and also we can say the sales of a product will be high if its quality is very good. So it is peculiar to survey these multisets where some sorting exists amid the attributes.

Recently the concept of multisets, soft groups and fuzzy soft goups are applied to lattice theory in $[1,2,13]$. J.Vimala proposed the notion of fuzzy lattice ordered group in [14] and thereby fuzzy $\ell-$ ideal was studied in [3]. Later on J.Vimala et.al.,[12] have formulate a decision making method that may be applied to many fields for solving problems containing uncertainties especially obtaining shortest route, convenient route among all routes by using fuzzy soft cardinality.

This paper is arranged in the following manner: In section 2, basic and necessary definitions concerning multisets and lattices are reviewed. In section 3 , we discuss the algebraic structures associated with lattice ordered multisets. In section 4 , we present an illustration that considering 0 and 1 as multiset depiction and identify their role in Boolean algebra over lattice ordered multisets(dual) while the order arising amid the attributes.

\section{Preliminaries}

In this section, we recall the necessary definitions concerning multisets and lattices.

Definition 2.1. [8] Let $X$ be any set. A multiset $M$ drawn from $X$ is represented by a function count $M$ or $C_{M}$ defined as $C_{M}: X \longrightarrow \mathbb{N}$ where $\mathbb{N}$ represents the set of all non-negative integers.

For each $x \in X, C_{M}(x)$ is the characteristic value of $x$ in $M$ and indicates the number of occurences of the element $x$ in $M$. A multiset $M$ is a set if $C_{M}(x)=0$ or $1 \forall x \in X$.

The word multiset often shortened to 'mset' abbreviates the term 'multiple membership set'.

$X$ is called the 'root' set or 'carrier' set or 'support' set of an mset $M$

Definition 2.2. [8] Let $M_{1}$ and $M_{2}$ be two multisets drawn from a set $X . M_{1}$ is a sub multiset of $M_{2}\left(M_{1} \subseteq M_{2}\right)$ if $C_{M_{1}}(x) \leqslant C_{M_{2}}(x)$ for all $x \in X . \quad M_{1}$ is a proper sub multiset of $M_{2}\left(M_{1} \subset M_{2}\right)$ if 
$C_{M_{1}}(x) \leqslant C_{M_{2}}(x)$ for all $x \in X$ and there exist at least one $x \in X$ such that $C_{M_{1}}(x)<C_{M_{2}}(x)$.

Definition 2.3. [8] Two multisets $M_{1}$ and $M_{2}$ are equal $\left(M_{1}=M_{2}\right)$ if $M_{1} \subseteq M_{2}$ and $M_{1} \supseteq M_{2}$.

Definition 2.4. [8] An multiset $M$ is empty if $C_{M}(x)=0$ for all $x \in X$.

Definition 2.5. [8] The union of two multisets $M_{1}$ and $M_{2}$ drawn from a set $X$ is an multiset $M$ denoted by $M=M_{1} \cup M_{2}$ such that $\forall x \in X, C_{M}(x)=\max$

$\left\{C_{M_{1}}(x), C_{M_{2}}(x)\right\}$.

Definition 2.6. [8] The intersection of two multisets $M_{1}$ and $M_{2}$ drawn from a set $X$ is an multiset $M$ denoted by $M=M_{1} \cap M_{2}$ such that $\forall x \in X, C_{M}(x)=\min$

$\left\{C_{M_{1}}(x), C_{M_{2}}(x)\right\}$

Definition 2.7. [8] Addition of two msets $M_{1}$ and $M_{2}$ drawn from a set $X$ results in a new mset $M=M_{1} \oplus M_{2}$ such that $\forall x \in X, C_{M}(x)=$ $C_{M_{1}}(x)+C_{M_{2}}(x)$

Definition 2.8. [8] Subtraction of two msets $M_{1}$ and $M_{2}$ drawn from a set $X$ results in a new mset $M=M_{1} \ominus M_{2}$ such that $C_{M}(x)=$ $\max \left\{C_{M_{1}}(x)-C_{M_{2}}(x), 0\right\}$

Definition 2.9. [8] Multiplication of two msets $M_{1}$ and $M_{2}$ drawn from a set $X$ results in a new mset $M=M_{1} \otimes M_{2}$ such that $\forall x \in$ $X, C_{M}(x)=C_{M_{1}}(x) \cdot C_{M_{2}}(x)$

Notation 2.10. [6] Let $M$ be an mset from $X$ and let $x$ appear $n$ times in $M$. We denote it by $x \in \in^{n} M . M=\left\{k_{1} / x_{1}, k_{2} / x_{2}, \ldots \ldots k_{n} / x_{n}\right\}$ also means that $M$ is an mset with $x_{1}$ appearing $k_{1}$ times, $x_{2}$ appearing $k_{2}$ times and so on. $[M]_{x}$ denotes the element $x$ belonging to the mset $M$ and $\left|[M]_{x}\right|$ denotes the cardinality of an element $x$ in $M$.

Definition 2.11. [10] Let $\mathfrak{p}=\left\{A_{1}, A_{2}, \ldots \ldots\right\}$ be a family of msets. The maximum mset $Z$ is defined by $C_{Z}(x)=\max _{A_{i} \in \mathfrak{p}} C_{A}(x)$ for all objects $x$. Then the complement of a mset $A$, denoted by $\bar{A}$ is defined as $\bar{A}=Z-A$ such that $C_{\bar{A}}(x)=C_{Z}(x)-C_{A}(x)$ for all objects $x$.

Hereinafter, $\max \{a, b\}$ and $\min \{a, b\}$ known as $a \vee b, a \wedge b$ respectively; $\mathrm{X}$ refers a root set of a mset that signifies to the lattice

Definition 2.12. [1] A multiset $M$ is called lattice(anti-lattice) ordered multiset whenever for the function $C_{M}: X \rightarrow \mathbb{N}, x \leqslant y$ implies $C_{M}(x) \leqslant C_{M}(y)\left[C_{M}(x) \geqslant C_{M}(y)\right]$ for all $x, y \in X$.

The word ' lattice ordered multiset' often shortened to ' $\ell$-mset' that abbreviates the term partially ordered multiple membership set in which each two-element submultiset has an infimum and a supremum.

Let us denote the elements of $\ell$-mset $\mathrm{M}$ as $p / x \leqslant q / y$ which means $x \leqslant y$ and $p \leqslant q$ where $C_{M}(x)=p, C_{M}(y)=q, p, q \in \mathbb{N}$

Example 2.13. Let $M=\{m / c, m+i / a, m+i+j / b, m+i / d, m+i+k / e\}$ with $i<j<k<m$ and $i, j, k, m \in \mathbb{N}$ be a multiset of gems annexed within a necklace. Here a,b,c,d,e depict platinum, diamond, coral, emerald, supphire respectively. Necklace is highly designed by supphire. Sorting of those elements is as delineated in figure 1 and whose tabular form is as given in below table

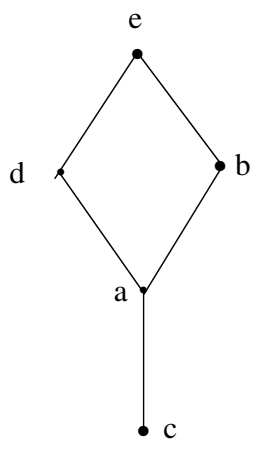

Figure 1
Table

\begin{tabular}{|l|c|c|c|c|}
\hline$C_{M}$ & $m$ & $m+i$ & $m+i+j$ & $m+i+k$ \\
\hline$a$ & 0 & 1 & 0 & 0 \\
$b$ & 0 & 0 & 1 & 0 \\
$c$ & 1 & 0 & 0 & 0 \\
$d$ & 0 & 1 & 0 & 0 \\
$e$ & 0 & 0 & 0 & 1 \\
\hline
\end{tabular}

\section{Algebraic structures associated with lattice ordered multisets}

In what follows, $C_{Z}(x)=\max _{A_{i} \in \mathfrak{p}} C_{A_{i}}(x), \forall x$ where $\mathfrak{p}=\left\{A_{1}, A_{2}, \cdots \cdots\right\}$ be a family of msets and $X$ be the root set of an mset that refers a lattice, unless otherwise stated.

$L M(X)$ - The collection of all $\ell-$ msets defined over $\mathrm{X}$

Proposition 3.1. Let $M_{1}$ and $M_{2}$ be two lattice ordered multisets over X. Then

(i) $C_{M_{1}}(x) \wedge\left[C_{M_{1}}(x) \vee C_{M_{2}}(x)\right]=C_{M_{1}}(x)$ for all $x$

(ii) $C_{M_{1}}(x) \vee\left[C_{M_{1}}(x) \wedge C_{M_{2}}(x)\right]=C_{M_{1}}(x)$ for all $x$

Proof. It is straight forward from the respective definitions

Proposition 3.2. $\left(L M(X), Z, \emptyset, \cup, \cap,,^{\prime}\right)$ is a De Morgan algebra

Proof. Since the De Morgan's laws

(i) $\left[C_{M}(x) \vee C_{N}(x)\right]^{\prime}=C_{M^{\prime}}(x) \wedge C_{N^{\prime}}(x)$ for all $\mathrm{x}$

(ii) $\left[C_{M}(x) \wedge C_{N}(x)\right]^{\prime}=C_{M^{\prime}}(x) \vee C_{N^{\prime}}(x)$ for all $\mathrm{x}$

hold good for each lattice ordered multisets $\mathrm{M}$ and $\mathrm{N}$ over $\mathrm{X}$, then it is a De Morgan algebra

Proposition 3.3. Let $\left(L M(X), Z, \emptyset, \cup, \cap,,^{\prime}\right)$ be a De Morgan algebra and $A, B$ be $\ell$-msets. Then

(i) $C_{A}(x) \leqslant C_{B}(x)$ if and only if $C_{A^{\prime}}(x) \geqslant C_{B^{\prime}}(x)$ and $C_{U^{\prime}}(x)=$ $C_{\emptyset}(x)$

(ii) $C_{A}(x) \neq C_{B}(x), C_{A}(x) \leqslant C_{B}(x) \Rightarrow$ either $C_{A}(x) \neq C_{A^{\prime}}(x)$ or $C_{B}(x) \neq C_{B^{\prime}}(x)$

Proof. (i) Suppose $C_{A}(x) \leqslant C_{B}(x)$, then

$C_{A}(x)=\left[C_{A}(x) \wedge C_{B}(x)\right]$ and $C_{A^{\prime}}(x)=\left[C_{A}(x) \wedge C_{B}(x)\right]^{\prime}=\left[C_{A^{\prime}}(x) \vee\right.$ $\left.C_{B^{\prime}}(x)\right] \Rightarrow C_{A^{\prime}}(x) \geqslant C_{B^{\prime}}(x)$.

Conversely, suppose $C_{U^{\prime}}(y) \neq C_{\emptyset}(y)$ and $E$ is an anyother $\ell$-mset. Then

$C_{E}(y)=\left[C_{E}(y) \wedge C_{U}(y)\right] \Rightarrow C_{E^{\prime}}(y)=\left[C_{E}(y) \wedge C_{U}(y)\right]^{\prime}=\left[C_{E^{\prime}}(y) \vee\right.$ $\left.C_{U^{\prime}}(y)\right\} \neq\left[C_{E^{\prime}}(y) \vee C_{\emptyset}(y)\right]=C_{E^{\prime}}(y)$ that is a contradiction. So, $C_{U^{\prime}}(x)=C_{\emptyset}(x)$

(ii) Suppose $C_{A}(x)=C_{A^{\prime}}(x)$ and $C_{B}(x)=C_{B^{\prime}}(x)$. By $(i), C_{A}(x) \leqslant$ $C_{B}(x)$ implies $C_{A}(x)=C_{A^{\prime}}(x) \geqslant C_{B^{\prime}}(x)=C_{B}(x)$, a contradiction

Proposition 3.4. Let $\left(L M(X), Z, \emptyset, \cup, \cap,^{\prime}\right)$ be a De Morgan algebra and $A, B$ be $\ell$-msets. Then $\left[C_{A}(z) \wedge C_{B^{\prime}}(z)\right]^{\prime}=C_{B}(z) \vee\left[C_{A^{\prime}}(z) \wedge\right.$ $\left.C_{B^{\prime}}(z)\right]$ if and only if $C_{B}(z) \wedge C_{B^{\prime}}(z) \leqslant C_{A}(z)$ for all $z \in X$

Proof. Assume for any $z \in X,\left[C_{A}(z) \wedge C_{B^{\prime}}(z)\right]^{\prime}=C_{B}(z) \vee\left[C_{A^{\prime}}(z) \wedge\right.$ $\left.C_{B^{\prime}}(z)\right]$

$\Rightarrow C_{A^{\prime}}(z) \vee C_{B}(z)=C_{B}(z) \vee\left[C_{A^{\prime}}(z) \wedge C_{B^{\prime}}(z)\right]$

Since $C_{A^{\prime}}(z) \leqslant C_{A^{\prime}}(z) \vee C_{B}(z)$, we have $C_{A^{\prime}}(z) \leqslant C_{B}(z) \vee\left[C_{A^{\prime}}(z) \wedge\right.$ $\left.C_{B^{\prime}}(z)\right]$

Then $\left\{C_{B}(z) \vee\left[C_{A^{\prime}}(z) \wedge C_{B^{\prime}}(z)\right]\right\}^{\prime}=C_{B^{\prime}}(z) \wedge\left[C_{A}(z) \vee C_{B}(z)\right] \leqslant$ $C_{A}(z)$.

Also $C_{B}(z) \wedge C_{B^{\prime}}(z) \leqslant\left[C_{A}(z) \vee C_{B}(z)\right] \wedge C_{B^{\prime}}(z)$ as $C_{B}(z) \leqslant$ $C_{A}(z) \vee C_{B}(z)$ 
Hence $C_{B}(z) \wedge C_{B^{\prime}}(z) \leqslant C_{A}(z)$

The second claim, assume $C_{B}(z) \wedge C_{B^{\prime}}(z) \leqslant C_{A}(z)$. It is equivalent to $C_{A^{\prime}}(z) \leqslant\left[C_{B^{\prime}}(z) \vee C_{B}(z)\right]$. $C_{A^{\prime}}(z) \vee C_{B}(z) \leqslant C_{B^{\prime}}(z) \vee C_{B}(z)$

Absorption, Commutativity, Distributivity imply

$C_{A^{\prime}}(z) \vee C_{B}(z) \leqslant\left\{C_{A^{\prime}}(z) \vee C_{B}(z)\right\} \wedge\left\{C_{B}(z) \vee C_{B^{\prime}}(z)\right\}$

$=\left[\left\{C_{A^{\prime}}(z) \wedge C_{B}(z)\right\} \vee\left\{C_{A^{\prime}}(z) \wedge C_{B^{\prime}}(z)\right\}\right]$

$\left[\left\{C_{B}(z) \wedge C_{B}(z)\right\} \vee\left\{C_{B}(z) \wedge C_{B^{\prime}}(z)\right\}\right]$

$=\left\{\left[\left\{C_{B}(z) \wedge\left\{C_{B}(z) \vee C_{B^{\prime}}(z)\right\}\right\} \vee\left\{C_{A^{\prime}}(z) \wedge C_{B}(z)\right\}\right] \vee\left\{C_{A^{\prime}}(z) \wedge C_{B^{\prime}}(z)\right.\right.$ $=\left\{\left[C_{B}(z) \vee\left\{C_{A^{\prime}}(z) \wedge C_{B}(z)\right\}\right] \vee\left\{C_{A^{\prime}}(z) \wedge C_{B^{\prime}}(z)\right\}\right\}$

$=\left\{C_{B}(z) \vee\left\{C_{A^{\prime}}(z) \wedge C_{B^{\prime}}(z)\right\}\right\}$

$C_{A^{\prime}}(z) \vee C_{B}(z) \leqslant\left\{C_{B}(z) \vee\left\{C_{A^{\prime}}(z) \wedge C_{B^{\prime}}(z)\right\}\right\}$

Moreover $C_{A^{\prime}}(z) \wedge C_{B^{\prime}}(z) \leqslant C_{A^{\prime}}(z)$ implies

$C_{B}(z) \vee\left[C_{A^{\prime}}(z) \wedge C_{B^{\prime}}(z)\right] \leqslant C_{A^{\prime}}(z) \vee C_{B}(z)$

By (1) and (2) $C_{B}(z) \vee\left[C_{A^{\prime}}(z) \wedge C_{B^{\prime}}(z)\right]=C_{A^{\prime}}(z) \vee C_{B}(z)$

Therefore $\left[C_{A}(z) \wedge C_{B^{\prime}}(z)\right]^{\prime}=C_{B}(z) \vee\left\{C_{A^{\prime}}(z) \wedge C_{B^{\prime}}(z)\right\}$

Proposition 3.5. Let $\left(\operatorname{LM}(X), Z, \emptyset, \cup, \cap,^{\prime}\right)$ be a De Morgan algebra and $\left[C_{A}(x) \wedge C_{B^{\prime}}(x)\right]^{\prime}=C_{B}(x) \vee\left[C_{A^{\prime}}(x) \wedge C_{B^{\prime}}(x)\right], \forall A, B \in L M(X)$. Then $\operatorname{LM}(X)$ is a Boolean algebra

Proof. For any $A, B \in L M(X), \quad\left[C_{A}(x) \wedge C_{B^{\prime}}(x)\right]^{\prime}=C_{B}(x) \vee$ $\left[C_{A^{\prime}}(x) \wedge C_{B^{\prime}}(x)\right]$ can be written as $C_{B}(x) \wedge C_{B^{\prime}}(x) \leqslant C_{A}(x)$ by above theorem. In particular, if $C_{A}(x)=C_{\emptyset}(x), \forall x \in X$, then $C_{B}(x) \wedge$ $C_{B^{\prime}}(x)=C_{\emptyset}(x)$ for every $B \in L M(X)$ and hence $C_{B}(x) \vee C_{B^{\prime}}(x)=$ $C_{Z}(x)$

Proposition 3.6. The only De Morgan algebras in which $\left[C_{A}(x) \wedge C_{B^{\prime}}(x)\right]^{\prime}=C_{B}(x) \vee\left[C_{A^{\prime}}(x) \wedge C_{B^{\prime}}(x)\right]$ holds are those that are Boolean algebras.

Proof. It follows from the above propositions

Example 3.7. Let $\left(L M(X), Z, \emptyset, \cup, \cap,^{\prime}\right)$ be a De Morgan algebra and let $Z=\{4 / x, 10 / y, 15 / z, 30 / w\}, A=\{0 / x, 10 / y, 15 / z, 30 / w\}$, $B=\{0 / x, 0 / y, 15 / z, 30 / w\}, \quad C=\{0 / x, 0 / y, 0 / z, 30 / w\}, \quad D=$ $\{0 / x, 0 / y, 0 / z, 0 / w\}, E=\{4 / x, 10 / y, 15 / z, 30 / w\}$. Since for every $P, Q \in L M(X)$ satisfy $\left\{C_{P}(a) \wedge C_{Q^{\prime}}(a)\right\}^{\prime}=C_{Q}(a) \vee\left[C_{P^{\prime}}(a) \wedge C_{Q^{\prime}}(a)\right]$ and it follows $C_{P}(a) \vee C_{P^{\prime}}(a)=C_{Z}(a)$ and $C_{P}(a) \wedge C_{P^{\prime}}(a)=C_{\emptyset}(a)$ for every $P \in L M(X), a \in X$

Example 3.8. Let $\mathscr{D}=\left(L M(X), Z, \emptyset, \cup, \cap,{ }^{\prime}\right)$ be a De Morgan algebra and let $Z=\{4 / x, 10 / y, 15 / z, 30 / w\}$, $A_{1}=\{2 / x, 5 / y, 8 / z, 12 / w\}, \quad A_{2}=\{3 / x, 6 / y, 7 / z, 10 / w\}$, $A_{3}=\{2 / x, 7 / y, 8 / z, 8 / w\}, A_{4}=\{1 / x, 4 / y, 5 / z, 15 / w\}$. Then $\mathscr{D}$ is not a boolean algebra because $A_{4} \cup\left(A_{1}^{\prime} \cap A_{4}^{\prime}\right) \neq\left(A_{1} \cap A_{4}^{\prime}\right)^{\prime}$

Proposition 3.9. $\left(L M(X), Z, \emptyset, \cup, \cap,^{\prime}\right)$ is a Kleene algebra

Proof. Let $M_{1}, M_{2} \in L M(X)$. Then $C_{M_{1}}(a) \wedge C_{M_{1}^{\prime}}(a) \leqslant C_{M_{2}}(a) \vee$ $C_{M_{2}^{\prime}}(a)$, for any $a \in X$. This shows that $\left(L M(X), Z, \emptyset, \cup, \cap,^{\prime}\right)$ is a Kleene algebra

\section{Application}

Like most of decision making problems, lattice ordered multisets based decision making involves the evaluation of all the objects which are decision alternatives.

Example 4.1. This is an example for score evaluation. Consider a problem for analysing the performance of students in a classroom and to increasing their knowledge. Thus making them ease for job seeking and as well as to excel them in irrespective of all the fields. Suppose that there are few kinds of scores: centum $\left(m_{5}\right)$, high score $\left(m_{4}\right)$, below average score $\left(m_{1}\right)$, average score $\left(m_{2}\right)$, good $\operatorname{score}\left(m_{3}\right)$ with the order $m_{1} \leqslant m_{2} \leqslant m_{3} \leqslant m_{4} \leqslant m_{5}$ are under evaluation according to following alternatives: repeated syllabi $\left(s_{1}\right)$, old syllabus with a few changes $\left(s_{2}\right)$, old syllabus with latest trends $\left(s_{3}\right)$, new syllabus $\left(s_{4}\right)$, new syllabus with global connects $\left(s_{5}\right)$, syllabus with real world applications $\left(s_{6}\right)$, advanced syllabus $\left(s_{7}\right)$. The lattice structure of syllabi from the root set $X$ is shown in fig 2 : $s_{1} \leqslant s_{4} \leqslant s_{5} \leqslant s_{7}$ and $s_{1} \leqslant s_{2} \leqslant s_{3} \leqslant s_{6} \leqslant s_{7}$.

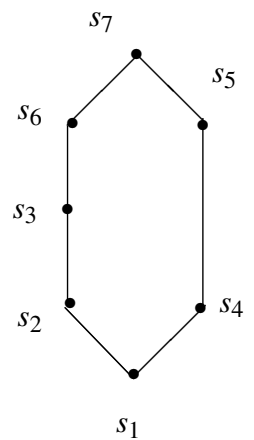

Figure 2

Suppose there are $\mathrm{T}$ number of students in the classroom

Step Formulate a new $k \in \mathbb{N}$ such that $k=\frac{T}{|i|},(i=1,2,3,4,5)$ and write 1 in particular $m_{i}$ if $\mathrm{k}$ number of students obtained the same $m_{i}$ score; otherwise 0 .

Table 1

\begin{tabular}{|l|c|c|c|c|c|}
\hline$C_{A}$ & $m_{1}$ & $m_{2}$ & $m_{3}$ & $m_{4}$ & $m_{5}$ \\
\hline$s_{1}$ & 0 & 0 & 1 & 0 & 1 \\
$s_{4}$ & 0 & 1 & 1 & 1 & 1 \\
$s_{5}$ & 1 & 0 & 1 & 1 & 0 \\
$s_{7}$ & 1 & 0 & 1 & 1 & 0 \\
\hline
\end{tabular}

Table 2

\begin{tabular}{|c|c|c|c|c|c|}
\hline$C_{B}$ & $m_{1}$ & $m_{2}$ & $m_{3}$ & $m_{4}$ & $m_{5}$ \\
\hline$s_{1}$ & 0 & 0 & 1 & 0 & 1 \\
$s_{2}$ & 0 & 1 & 0 & 1 & 1 \\
$s_{3}$ & 0 & 1 & 0 & 1 & 1 \\
$s_{6}$ & 1 & 0 & 1 & 1 & 0 \\
$s_{7}$ & 1 & 0 & 1 & 1 & 0 \\
\hline
\end{tabular}

If $s_{j, k, l}, s_{j, k, h}$ stand for the lowest and highest score which are obtained by at least $\mathrm{k}$ number of students for the syllabi $s_{j},(j=1,2,3,4,5,6,7)$ respectively, then $s_{1, k, l}=m_{3}, s_{2, k, l}=$ $m_{2} s_{3, k, l}=m_{2}, s_{4, k, l}=m_{2}, s_{5, k, l}=m_{1}, s_{6, k, l}=m_{1}, s_{7, k, l}=m_{1}$ and $s_{1, k, h}=m_{5}, s_{2, k, h}=m_{5} s_{3, k, h}=m_{5}, s_{4, k, h}=m_{5}, s_{5, k, h}=m_{4}, s_{6, k, h}=$ $m_{4}, s_{7, k, h}=m_{4}$.

In this manner, we arrange $C_{A}\left(s_{7}\right) \leqslant C_{A}\left(s_{5}\right) \leqslant C_{A}\left(s_{4}\right) \leqslant C_{A}\left(s_{1}\right)$ and $C_{B}\left(s_{7}\right) \leqslant C_{B}\left(s_{6}\right) \leqslant C_{B}\left(s_{3}\right) \leqslant C_{B}\left(s_{2}\right) \leqslant C_{B}\left(s_{1}\right)$.

These relations would combine to imply that $A$ and $B$ are anti-lattice ordered multisets. 
Table 3

\begin{tabular}{|l|c|c|c|c|c|}
\hline$C_{A^{\prime}}$ & $m_{1}$ & $m_{2}$ & $m_{3}$ & $m_{4}$ & $m_{5}$ \\
\hline$s_{1}$ & 1 & 1 & 0 & 1 & 0 \\
$s_{4}$ & 1 & 0 & 0 & 0 & 0 \\
$s_{5}$ & 0 & 1 & 0 & 0 & 1 \\
$s_{7}$ & 0 & 1 & 0 & 0 & 1 \\
\hline
\end{tabular}

Table 4

\begin{tabular}{|l|c|c|c|c|c|}
\hline$C_{B^{\prime}}$ & $m_{1}$ & $m_{2}$ & $m_{3}$ & $m_{4}$ & $m_{5}$ \\
\hline$s_{1}$ & 1 & 1 & 0 & 1 & 0 \\
$s_{2}$ & 1 & 0 & 1 & 0 & 0 \\
$s_{3}$ & 1 & 0 & 1 & 0 & 0 \\
$s_{6}$ & 0 & 1 & 0 & 0 & 1 \\
$s_{7}$ & 0 & 1 & 0 & 0 & 1 \\
\hline
\end{tabular}

Step Construct table and find $A \wedge B^{\prime}=\left\{m \wedge n: m \in A, n \in B^{\prime}\right\}$

Table 5

\begin{tabular}{|l|c|c|c|c|c|}
\hline$C_{A} \wedge C_{B^{\prime}}$ & $m_{1}$ & $m_{2}$ & $m_{3}$ & $m_{4}$ & $m_{5}$ \\
\hline$s_{1} \wedge s_{1}$ & 0 & 0 & 0 & 0 & 0 \\
$s_{1} \wedge s_{2}$ & 0 & 0 & 1 & 0 & 0 \\
$s_{1} \wedge s_{3}$ & 0 & 0 & 1 & 0 & 0 \\
$s_{1} \wedge s_{6}$ & 0 & 0 & 0 & 0 & 1 \\
$s_{1} \wedge s_{7}$ & 0 & 0 & 0 & 0 & 1 \\
$s_{4} \wedge s_{1}$ & 0 & 1 & 0 & 1 & 0 \\
$s_{4} \wedge s_{2}$ & 0 & 0 & 1 & 0 & 0 \\
$s_{4} \wedge s_{3}$ & 0 & 0 & 1 & 0 & 0 \\
$s_{4} \wedge s_{6}$ & 0 & 1 & 0 & 0 & 1 \\
$s_{4} \wedge s_{7}$ & 0 & 1 & 0 & 0 & 1 \\
$s_{5} \wedge s_{1}$ & 1 & 0 & 0 & 1 & 0 \\
$s_{5} \wedge s_{2}$ & 1 & 0 & 1 & 0 & 0 \\
$s_{5} \wedge s_{3}$ & 1 & 0 & 1 & 0 & 0 \\
$s_{5} \wedge s_{6}$ & 0 & 0 & 0 & 0 & 0 \\
$s_{5} \wedge s_{7}$ & 0 & 0 & 0 & 0 & 0 \\
$s_{7} \wedge s_{2}$ & 1 & 0 & 1 & 0 & 0 \\
$s_{7} \wedge s_{3}$ & 1 & 0 & 1 & 0 & 0 \\
$s_{7} \wedge s_{6}$ & 0 & 0 & 0 & 0 & 0 \\
$s_{7} \wedge s_{7}$ & 0 & 0 & 0 & 0 & 0 \\
\hline
\end{tabular}

StepAfter constructing a table for $\left[C_{A}(x) \wedge C_{B^{\prime}}(x)\right]^{\prime}$ and pick the maximum value of $\sum_{i, j} s_{i} \wedge s_{j}$ (column sum)

Table 6

\begin{tabular}{|l|c|c|c|c|c|}
\hline Score & $m_{1}$ & $m_{2}$ & $m_{3}$ & $m_{4}$ & $m_{5}$ \\
\hline Total & 14 & 16 & 11 & 17 & 15 \\
Rank & 4th & 2nd & 5 th & 1 st & 3 rd \\
\hline
\end{tabular}

The utmost priority goes to ' $m_{4}$ '

6.1 To find the most obtained score

Another side for Boolean algebra over $\ell$-mset Table 7

\begin{tabular}{|l|c|c|c|c|c|}
\hline$C_{A^{\prime}} \wedge C_{B^{\prime}}$ & $m_{1}$ & $m_{2}$ & $m_{3}$ & $m_{4}$ & $m_{5}$ \\
\hline$s_{1} \wedge s_{1}$ & 1 & 1 & 0 & 1 & 0 \\
$s_{1} \wedge s_{2}$ & 1 & 0 & 0 & 0 & 0 \\
$s_{1} \wedge s_{3}$ & 1 & 0 & 0 & 0 & 0 \\
$s_{1} \wedge s_{6}$ & 0 & 1 & 0 & 0 & 0 \\
$s_{1} \wedge s_{7}$ & 0 & 1 & 0 & 0 & 0 \\
$s_{4} \wedge s_{1}$ & 1 & 0 & 0 & 0 & 0 \\
$s_{4} \wedge s_{2}$ & 1 & 0 & 0 & 0 & 0 \\
$s_{4} \wedge s_{3}$ & 1 & 0 & 0 & 0 & 0 \\
$s_{4} \wedge s_{6}$ & 0 & 0 & 0 & 0 & 0 \\
$s_{4} \wedge s_{7}$ & 0 & 0 & 0 & 0 & 0 \\
$s_{5} \wedge s_{1}$ & 0 & 1 & 0 & 0 & 0 \\
$s_{5} \wedge s_{2}$ & 0 & 0 & 0 & 0 & 0 \\
$s_{5} \wedge s_{3}$ & 0 & 0 & 0 & 0 & 0 \\
$s_{5} \wedge s_{6}$ & 0 & 1 & 0 & 0 & 1 \\
$s_{5} \wedge s_{7}$ & 0 & 1 & 0 & 0 & 1 \\
$s_{7} \wedge s_{2}$ & 0 & 0 & 0 & 0 & 0 \\
$s_{7} \wedge s_{3}$ & 0 & 0 & 0 & 0 & 0 \\
$s_{7} \wedge s_{6}$ & 0 & 1 & 0 & 0 & 1 \\
$s_{7} \wedge s_{7}$ & 0 & 1 & 0 & 0 & 1 \\
\hline
\end{tabular}

StepSimplify the constructed values of $C_{B}(x) \vee\left[C_{A^{\prime}}(x) \wedge C_{B^{\prime}}(x)\right]$ and find the maximum value of $\sum_{i, j} s_{j} \vee\left[s_{i} \wedge s_{j}\right]$

Table 8

\begin{tabular}{|l|c|c|c|c|c|}
\hline Score & $m_{1}$ & $m_{2}$ & $m_{3}$ & $m_{4}$ & $m_{5}$ \\
\hline$\sum_{i, j} s_{1} \vee\left[s_{i} \wedge s_{j}\right]$ & 6 & 8 & 19 & 1 & 19 \\
$\sum_{i, j} s_{2} \vee\left[s_{i} \wedge s_{j}\right]$ & 6 & 19 & 0 & 19 & 19 \\
$\sum_{i, j} s_{3} \vee\left[s_{i} \wedge s_{j}\right]$ & 6 & 19 & 0 & 19 & 19 \\
$\sum_{i, j} s_{6} \vee\left[s_{i} \wedge s_{j}\right]$ & 19 & 8 & 19 & 19 & 4 \\
$\sum_{i, j} s_{7} \vee\left[s_{i} \wedge s_{j}\right]$ & 19 & 8 & 19 & 19 & 4 \\
\hline Total & 56 & 62 & 57 & 77 & 65 \\
\hline Rank & 5th & 3rd & 4th & 1 st & 2nd \\
\hline
\end{tabular}

From this, $m_{1} \leq m_{3} \leq m_{2} \leq m_{5} \leq m_{4}$ and $\left[C_{A}(x) \wedge C_{B^{\prime}}(x)\right]^{\prime}=$ $C_{B}(x) \vee\left[C_{A^{\prime}}(x) \wedge C_{B^{\prime}}(x)\right]$

Thus the systems are in Boolean algebraic structure and the most obtained score is ' $m_{4}$ (high score)

6.2 To find the favourite syllabi

Step Construct the decision matrix

$\begin{array}{lllll}m_{1} & m_{2} & m_{3} & m_{4} & m_{5}\end{array}$

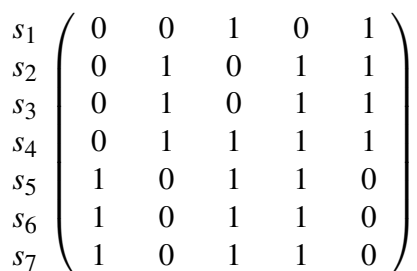

Step Obtained the below matrix by $a_{i j}^{\prime}=\frac{a_{i j}}{\sum_{i} a_{i j}}$

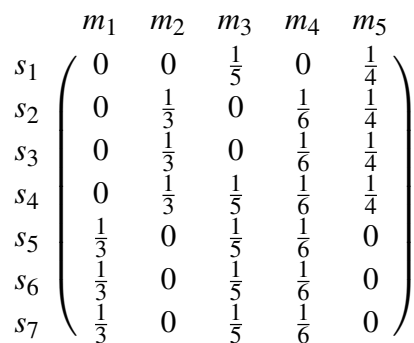

Step Compute relative weight $w_{t}$ for each alternatives $s_{j}$ by $w_{t}=\frac{\sum_{j=1}^{7} a_{i j}}{|i|},(i=1,2,3,4,5)$ where $\sum_{t=1}^{7} w_{t}=1$

So, $w_{1}=0.1, w_{2}=0.15, w_{3}=0.145, w_{4}=0.195, w_{5}=$ 
$0.14, w_{6}=0.13, w_{7}=0.14$

Step $a_{i j}^{*}=\frac{a_{i j}^{\prime}}{\max \left(a_{i j}\right)}$ is shown in following matrix

\begin{tabular}{|c|c|c|c|c|}
\hline$m_{1}$ & $m_{2}$ & $m_{3}$ & $m_{4}$ & $m_{5}$ \\
\hline 0 & 0 & 0.8 & 0 & 1 \\
\hline 0 & 1 & 0 & 0.5 & 0.75 \\
\hline 0 & 1 & 0 & 0.5 & 0.75 \\
\hline 0 & 1 & 0.6 & 0.5 & 0.75 \\
\hline 1 & 0 & 0.6 & 0.5 & 0 \\
\hline 1 & 0 & 0.6 & 0.5 & 0 \\
\hline 1 & 0 & 0.6 & 0.5 & 0 \\
\hline
\end{tabular}

Step Next compute $\overline{a_{i j}}=w_{t} a_{i j}^{*}$

\begin{tabular}{|c|c|c|c|c|c|}
\hline & $m_{1}$ & $m_{2}$ & $m_{3}$ & $m_{4}$ & $m_{5}$ \\
\hline$s_{1}$ & 0 & 0 & 0.02 & 0 & 0.025 \\
\hline$s_{2}$ & 0 & 0.15 & 0 & 0.075 & 0.113 \\
\hline$s_{3}$ & 0 & 0.145 & 0 & 0.073 & 0.108 \\
\hline$s_{4}$ & 0 & 0.195 & 0.117 & 0.098 & 0.146 \\
\hline$s_{5}$ & 0.14 & 0 & 0.08 & 0.07 & 0 \\
\hline$s_{6}$ & 0.13 & 0 & 0.08 & 0.065 & 0 \\
\hline$s_{7}$ & 0.14 & 0 & 0.08 & 0.07 & 0 \\
\hline
\end{tabular}

Step Pick out the maximum value in the row sum

\begin{tabular}{|c|c|c|c|c|c|c|c|}
\hline Score & $s_{1}$ & $s_{2}$ & $s_{3}$ & $s_{4}$ & $s_{5}$ & $s_{6}$ & $s_{7}$ \\
\hline Total & 0.045 & 0.34 & 0.33 & 0.556 & 0.29 & 0.275 & 0.29 \\
Rank & 7 th & 2nd & 3rd & 1 st & 4th & 6th & 4th \\
\hline
\end{tabular}

6.3 To find the syllabi which takes minimum time

Step Calculate $\wedge\left[\begin{array}{c}\sum_{j} s_{i j} \\ \bigvee s_{i}\end{array}\right]$ for $s_{i} i=1,2,3,4,5$ since $s_{1}$ and $s_{7}$ are the least upper bound and greatest lower bound of both the chains $s_{1} \leqslant s_{4} \leqslant s_{5} \leqslant s_{7}$ and $s_{1} \leqslant s_{2} \leqslant s_{3} \leqslant s_{6} \leqslant s_{7}$.

$$
\begin{aligned}
\wedge & {\left[\begin{array}{c}
\sum_{j} s_{i j} \\
\frac{j}{V} s_{i}
\end{array}\right]=\bigwedge\left\langle\frac{0.34}{0.15}, \frac{0.33}{0.145}, \frac{0.556}{0.195}, \frac{0.29}{0.14}, \frac{0.275}{0.13}\right\rangle } \\
& =\bigwedge\langle 2.266,2.275,2.851,2.071,2.115\rangle \\
& =2.071 \\
& =s_{5}
\end{aligned}
$$

Thus the syllabi ' $s_{5}$ ' is most likely hold minimum time

\section{Conclusion}

In this paper, we introduced some algebraic structures De Morgan algebra, Boolean algebra, Kleene algebra associated with $\ell$-msets. To extend this work, one can survey the other algebraic structures of $\ell$-msets such as Heyting algebra and Stone algebras. Moreover we have formulate a decision making method that may be applied to many fields for solving problems containing uncertainties and this attitude could be alternative comprehensive to solve pertinent problems in the future.

\section{Acknowledgement}

The authors would like to express their sincere thanks to the reviewers and Editor-in-Chief of the journal, for their valuable comments and suggestions.

The research is partially supported by the Department of Science and Technology (DST-PURSE), India [Grant no. 10815]

\section{References}

[1] Anusuya Ilamathi V.S., Vimala J. (2018) Multi-criteria Decision Making on Lattice Ordered Multisets. In: Thampi S., Mitra S., Mukhopadhyay J., Li KC., James A., Berretti S. (eds) Intelligent Systems Technologies and Applications. ISTA 2017. Advances in Intelligent Systems and Computing, vol 683. Springer, Cham

[2] J.AROCKIA REETA, J.VimalA, A study on distributive and modular lattice ordered fuzzy soft group and its duality, Applied Mathematics Journal of Chinese Universities-Springer, 31(4) (2016) 491-502
[3] P.Bharathi, J.Vimala et.al., Some Contributions of Congruence Relations on Lattice of Fuzzy $\ell$-ideals, Applied Mathematics \& Information Sciences II No. 2 (2017) 509-513

[4] G.BIRKHOFF, Lattice Theory. 3rd ed., American Mathematical Society. Providence, RL, 1967

[5] K.P.GIRISH, S.J.JOHN, Relations and functions in the multiset context, Information sciences 179(2009) 758-768.

[6] K.P.GIRISH, S.J.JOHN, General relations between partially ordered multisets and their chains and antichains, Mathematical Communications, 14(2) (2009a) 193-206.

[7] G.GRATZER,General Lattice Theory, 2nd Edition, Birkhauser Verlag, Basel-Boston, Berlin, 2003.

[8] S.P.JENA, S.K.GHOSH, B.K.TRIPATHY, On the theory of bags and lists, Information sciences 132 (2001) 241-254.

[9] R.R.YAGER, On the theory of bags, International Journal of General Systems, 13(1) (1986), 23-37.

[10] D. Singh, A. M. Ibrahim, T. Yohana, J. N. Singh, Complementation in Multiset Theory, International Mathematical Forum, 6(38), (2011), 1877 - 1884

[11] A.SYROPOULOS, Mathematics of Multisets, In: Multiset Processing, C.Calude, G.Paun, G.Rozenberg, and A.Salomaa, eds., Lecture Notes in Computer Science. Springer-Verlag, Berlin, 2235 (2001), 347-358.

[12] J.Vimala, J.Arockia REETA, VS. AnusuYa Ilamathi, $A$ Study On Fuzzy Soft Cardinality In Lattice Ordered Fuzzy Soft Group And Its Application In Decision Making Problems, Journal of Intelligent and Fuzzy Systems (Accepted)

[13] L.ViJaYAlaKSHMI, J.Vimala On Lattice Ordered Soft Groups, International Journal of Pure and Applied Mathematics, 1(112) (2017), $47-55$

[14] J.VIMALA, Fuzzy Lattice Ordered Group International Journal of Scientific \& Engineering Research, 5(9) (2014) 58-60

[15] WAYNE.D.Blizard, Multiset Theory, Notre Dame Journal of Formal Logic 30(1) (1989a), 36-66. 